\title{
Mise au point d'une méthode de mesure de la concentration totale en cellules de Brevibacterium linens à la surface d'un fromage à pâte molle et croûte lavée
}

\author{
A Oumer, MN Leclercq-Perlat, JL Bergère, G Corrieu
}

\author{
Laboratoire de génie et de microbiologie des procédés alimentaires, \\ Inra, 78850 Thiverval-Grignon, France
}

(Reçu le 16 novembre 1995 ; accepté le 13 mars 1996)

\begin{abstract}
Résumé - Une méthode de mesure de la concentration totale en cellules de Brevibacterium linens ATCC 9175 en surface d'un fromage est développée. Dans un premier temps, la mise au point de cette méthode est réalisée dans un milieu liquide, puis, appliquée au cas du fromage. Cette méthode est fondée sur la solubilisation des pigments cellulaires par le méthanol. Les meilleures conditions d'extraction correspondent à une incubation de $1 \mathrm{~h}$ à $25^{\circ} \mathrm{C}$ à partir du culot cellulaire provenant d'une culture en milieu liquide. En présence de fromage, il est nécessaire d'éliminer au préalable les matières grasses solubilisées par le méthanol en laissant l'extrait méthanolique $1 \mathrm{~h}$ à $4^{\circ} \mathrm{C}$. Une mesure d'absorbance à $450 \mathrm{~nm}$ détermine le niveau de pigmentation. La pigmentation de $B$ linens ATCC 9175 est indépendante de la lumière, de la concentration en chlorure de sodium et du milieu de culture utilisé (liquide ou fromage). Dans le cas de cultures en milieu liquide de composition proche de celle d'un caillé, les mesures d'absorbance à $450 \mathrm{~nm}$ traduisant la quantité de pigments sont comparées aux résultats obtenus par d'autres méthodes de mesure de la concentration cellulaire totale (quantité de matière sèche et absorbance à $650 \mathrm{~nm}$ ). Bien que les corrélations linéaires entre quantité de matière sèche et quantité de pigments soient tout à fait correctes pour un essai donné, elles ne sont pas généralisables et de ce fait ne peuvent être utilisées. En revanche, la corrélation entre absorbance à $650 \mathrm{~nm}$ et quantité de pigments a une linéarité satisfaisante $\left(R^{2} \geq 0,90\right)$ et est indépendante de l'essai considéré. La mesure de la quantité de pigments par absorbance à $450 \mathrm{~nm}$ peut être utilisée comme moyen de détermination rapide de la concentration totale en cellules de $B$ linens, ATCC 9175, de fromages expérimentaux à flore d'affinage définie. Elle serait applicable à d'autres souches de $B$ linens dont la pigmentation est stable. Dans le cas de mélanges microbiens plus complexes, tels que ceux présents en surface des fromages industriels, cette méthode, ne peut être utilisée que si les autres bactéries présentes ne sont pas pigmentées.
\end{abstract}

Brevibacterium linens / concentration cellulaire / quantité de pigments cellulaires / extraction méthanolique / fromage à pâte molle et croûte lavée

Summary - Quantitative method for enumeration of Brevibacterium linens cells in bacterial surface-ripened cheese. A method for enumeration of B linens cells ATCC 9175 in surface of bacterial surface-ripened cheese by estimation of pigment quantity was developed. In first time, this method was realized in liquid medium, after that applied in cheese. The cellular pigments of $B$ linens 
were completely solubilized by methanol. The best conditions of solubility were $1 \mathrm{~h}$ at $25^{\circ} \mathrm{C}$ from cell liquid culture. In cheese, it is necessary to eliminate the fat content by the methanol solution by standing it $1 \mathrm{~h}$ at $4{ }^{\circ} \mathrm{C}$ before filtration. The cellular pigmentation was measured by absorbance at $450 \mathrm{~nm}$. Absorbance measure at $450 \mathrm{~nm}$ was equivalent to level of pigmentation. The pigmentation of $\mathrm{B}$ linens ATCC 9175 was independent of light, of sodium chloride concentration and growth medium (liquid or cheese). In liquid medium with similar composition of curd, the cell dry weight matter and turbidimetric absorbance at $650 \mathrm{~nm}$ were chosen as reference methods. The dry weight method could not be used because the correlation established between pigment quantity and dry weight were different for each test. The correlation established between turbidimetric absorbance at $650 \mathrm{~nm}$ and pigment quantity was satisfying $\left(R^{2} \geq 0.90\right)$ and independent of the test considered. The pigment quantity method by absorbance at $450 \mathrm{~nm}$ could be used for rapid determination of total cell concentration of $\mathrm{B}$ linens ATCC 9175 in experimental bacterial surface-ripened cheeses during ripening. This method would be applicable to stable pigmentation B linens strains. In the case of microbial mixtures like that existing in the surface of industrial cheese, this pigment quantity method could be used only if the other bacteria did not produce pigments.

\section{Brevibacterium linens / cellular concentration / cell pigment quantity / extraction by methanol / bacterial surface-ripened cheese}

\section{INTRODUCTION}

Selon Lenoir (1991), les fromages à pâte molle et à croûte lavée, comme tout produit alimentaire, doivent répondre aux deux exigences suivantes : hygiène et goût. Or, l'ałfinage de ces fromages est un phénomène complexe et dynamique. En effet, il est caractérisé par le développement en surface d'une flore microbienne composée d'une grande variété d'espèces appartenant à des groupes différents (moisissures, levures, bactéries). Ces groupes microbiens représentent des acteurs essentiels dans la détermination de la typicité des fromages (Lenoir et al, 1985). En effet, ils contribuent selon leur nature et suivant les caractéristiques du fromage à la détermination de la texture, de l'arôme et du goût du produit final. Ces micro-organismes possèdent des potentialités métaboliques variées, telles que, pour les levures, assimilation de l'acide lactique et du lactose, activités d'estérification et de dégradation de protéines et de peptides et activités lipolytiques, pour les bactéries activités protéolytiques allant jusqu'à la dégradation des acides aminés et activités lipolytiques (Lenoir et al, 1983). La population microbienne viable atteint généralement $10^{\circ}$ à
$10^{10}$ germes viables par gramme de croûte, parfois plus. Il est généralement admis qu'à partir d'un certain stade, elle évolue relativement peu au cours de l'affinage et qu'elle est affectée par une mortalité importante. Cependant, l'état physiologique et les activités biologiques de cette population sont mal connus. S'agit-il d'une population dont les cellules sont restées viables ou existe$t$-il un renouvellement des cellules au fur et à mesure qu'une partie d'entre elles meurent ? En outre, l'équilibre entre les différentes espèces est sûrement en constante évolution. II est fonction des particularités propres à chaque groupe microbien et de ce fait, dépend de facteurs physico-chimiques $\left(\mathrm{a}_{\mathrm{w}}, \mathrm{pH}\right.$, composition...), des conditions d'environnement (température, humidité relative, atmosphère...) et des interactions entre souches (coopération, inhibition...).

Afin d'améliorer la fabrication fromagère, il faut connaître et maîtriser les conditions physico-chimiques et microbiologiques de leur affinage. Cela nécessite une meilleure connaissance du comportement, du développement, de l'état physiologique et de l'activité biologique des populations mixtes de micro-organismes constituant la flore de surface. Pour étudier cela, nous avons 
entrepris de fabriquer aseptiquement, à partir d'un lait microfiltré et pasteurisé, des fromages expérimentaux de type pâte molle-croûte lavée en limitant à deux le nombre de souches d'affinage (Geotrichum candidum, Brevibacterium linens). Cela implique de quantifier la concentration en cellules "viables "et « non viables »en différenciant chacune des deux espèces.

La concentration totale en levures ( $G$ candidum, Debaryomyces hansenii...) à la surface des fromages est déterminée par une méthode de numération à l'aide d'un compteur de particules de type Coulter après élimination préalable des constituants du fromage par transparisation (Leclercq-Perlat et al, 1995). Les différentes espèces de levures présentes sont différenciables quand la différence de leur diamètre moyen est supérieure à $1 \mu \mathrm{m}$. Les cellules bactériennes de diamètre équivalent inférieur à 2 um (ce qui est généralement le cas chez $B$ linens) ne peuvent être comptées avec ce type d'appareil car des particules de fromages, de diamètre inférieur ou égal à $1,8 \mu \mathrm{m}$, restent insolubles après transparisation.

En outre, après le traitement de transparisation, la mesure de la concentration bactérienne totale (viables et non viables) s'avère impossible quelle que soit la méthode utilisée : compteur Coulter, comptage direct des cellules au microscope, cytométrie de flux (Hadley et al, 1985) ou mesure optique (néphélométrie ou turbidimétrie) (Bourgeois et Mafart, 1991). En effet, la majorité des cellules bactériennes sont détruites par le réactif de transparisation (Driehuis et Teernstra, 1992) ou vidées de leur contenu cellulaire (Kouomegne et al, 1984). De plus, des constituants du réactif de transparisation peuvent pénétrer et/ou adhérer aux cellules (Miozzari et al, 1978).

Dagonneau (1979) a proposé d'évaluer la concentration cellulaire totale de cultures pures de $B$ linens en mesurant celle d'un hydrocarbure spécifique de leur enveloppe cellulaire par chromatographie en phase gazeuse. Cependant, cet auteur montre que la concentration en hydrocarbure est difficilement corrélée à la biomasse totale obtenue par mesure du poids sec. II explique cela par une trop faible concentration de l'hydrocarbure dans l'enveloppe cellulaire. De plus, l'application au cas des fromages s'est avérée impossible.

En revanche, le dosage des pigments orange de $B$ linens pourrait peut-être servir à mesurer, dans les fromages, la concentration totale en cellules de cette bactérie, y compris celles qui se sont lysées. La couleur jaune-orange de $B$ linens est relativement stable (Khol et al, 1983). Elle est en rapport avec le changement de couleur caractéristique de l'orange au rouge carmin des cellules de cette bactérie en présence d'alcali forts (Grecz et Dack, 1961 ; Jones et al, 1973). Selon Jones et al (1973), Fautz et Reinchenbach (1980) et Khol et al (1983), elle est due à des pigments se trouvant dans la membrane des cellules et appartenant à la famille des caroténoïdes. Dans le cas de la souche ATCC 9175, Khol et al (1983) ont montré l'existence et la structure de trois caroténoïdes de type aromatique responsables de la pigmentation des cellules et du changement caractéristique de couleur des colonies provoqué par les alcali forts.

Cependant, Mulder et al (1966) et Crombach (1974) ont constaté que certaines souches de $B$ linens ne se pigmentent pas si elles se développent à l'obscurité alors que d'autres sont insensibles à ce facteur. De plus, Grecz et Dack (1961), Mulder et al (1966) et Crombach (1974) ont observé que l'intensité de la couleur de $B / i$ nens est fonction de la souche considérée et, pour une même souche, de la composition du milieu de culture, en particulier de la concentration en $\mathrm{NaCl}$ et du $\mathrm{pH}$. En dépit de ces variations, la réaction de coloration à la potasse $(\mathrm{KOH}, 5 \mathrm{~N})$ a toujours lieu. Mais ces différents auteurs n'ont pas vérifié si ces facteurs avaient une influence sur la quantité de pigments produits. 
L'étude des pigments se fait toujours après extraction à l'aide de solvants (méthanol, acétone, toluène) utilisés purs ou en mélange. Ainsi, pour extraire les pigments des cellules de $B$ linens, Jones et al (1973) utilisent le méthanol pur à $65^{\circ} \mathrm{C}$, Fautz et Reinchenbach (1980) utilisent un mélange acétone-toluène et plus récemment Khol et al (1983) préconisent l'emploi de méthanol pur à température ambiante.

L'objectif de notre travail est de mettre au point une méthode permettant de quantifier la concentration totale en $B$ linens á la surface d'un fromage, par mesure de la quantité de pigments des cellules. II nous a paru utile de commencer l'étude en utilisant un milieu liquide (culture pure de $B$ linens) de composition se rapprochant à celle d'un caillé. Par la suite, nous appliquons cette méthode au fromage.

\section{MATÉRIEL ET MÉTHODES}

\section{Souches et conditions de culture}

\section{Souche utilisée}

La souche de $B$ linens choisie, ATCC 9175, a été isolée du camembert et se pigmente aussi bien à la lumière qu'à l'obscurité. Comme toutes les autres souches de cette espèce, elle tolère de fortes concentrations en sel (jusqu'à $15 \%$ ) (Crombach, 1974). Sa température optimale de croissance est de $25^{\circ} \mathrm{C}$ et son pH optimal est voisin de la neutralité ou légèrement alcalin (Lecocq et Gueguen, 1994). En surface des fromages, les moisissures et/ou les levures comme $G$ candidum métabolisent le lactate et entraînent une remontée du $\mathrm{pH}$ du caillé, ce qui permet à $B$ linens de s'implanter (Boyaval et Desmazeaud, 1983). Les cellules de $B$ linens apparaissent après 10 à 15 jours d'affinage à $13{ }^{\circ} \mathrm{C}$ et $95 \%$ d'humidité relative. Dans le cas de la souche ATCC 9175, elles sont opaques et de couleur orange.

\section{Cultures en milieu liquide}

Les cultures en milieu liquide sont réalisées dans un bouillon de composition proche de celle du fromage. Soit, par litre d'eau : lactate de sodium (20 g ; Prolabo), extrait autolytique de levure
( $5 \mathrm{~g}$; Biokar), peptone trypsique de caséine de type bio-trypcase ( $15 \mathrm{~g}$; Bio-Mérieux), chlorure de sodium ( $\mathrm{NaCl}$ ) ( 0 à $50 \mathrm{~g}$; Prolabo), potassium dihydrogénophosphate $\left(\mathrm{KH}_{2} \mathrm{PO}_{4}\right)(5 \mathrm{~g}$; Prolabo). Après autoclavage $\left(121^{\circ} \mathrm{C}, 20 \mathrm{~min}\right)$, les cultures sont ensemencées à raison de $1 \%$ avec une préculture de $B$ linens réalisée dans le même milieu et incubée sous une agitation à $25^{\circ} \mathrm{C}$ pendant $36 \mathrm{~h}$. Puis, les cultures sont incubées à $25^{\circ} \mathrm{C}$ sous agitation de 250 tours/minute (agitateur LSL Biolafitte, France).

Chaque culture de $B$ linens est faite dans un erlenmeyer de $5 \mathrm{~L}$ contenant $2,5 \mathrm{~L}$ de milieu à $\mathrm{pH} 7$ et $1 \%$ de $\mathrm{NaCl}$. Après autoclavage et ensemencement, la culture est incubée pendant 8 jours. Des prélèvements stériles de $60 \mathrm{~mL}$ sont effectués toutes les 4 heures pendant les 4 premiers jours d'incubation et puis toutes les 6 heures jusqu'au $8^{\mathrm{e}}$ jour. Sur chaque prélèvement, sont réalisées les analyses suivantes : dénombrement en cellules viables, mesure de l'absorbance à $650 \mathrm{~nm}$, mesure de la quantité de la matière sèche et mesure de la quantité de pigments. Chaque essai a été réalisé en double.

\section{Cultures en surface des fromages}

Dans ce cas, les fromages sont fabriqués aseptiquement dans une enceinte préalablement stérilisée et thermostatée à $30^{\circ} \mathrm{C}$. Le lait utilisé est un lait écrémé microfiltré (laboratoire de recherches de technologie laitière, Inra, Rennes). II est reconstitué à $10 \%$ et additionné de crème à $35 \%$ de sorte que sa concentration en matière grasse soit égale à celle d'un lait entier. Après reconstitution, le lait est pasteurisé à $72^{\circ} \mathrm{C}$ pendant $1 \mathrm{~min} 30$ secondes. II est ensuite ensemencé à $1 \%$ par le levain lactique (Lactococcus lactis Prot' (souche 1128 ) et Lactococcus lactis Prot (souche 1130)) et le levain d'affinage. Ce dernier est constitué de $B$ linens ATCC 9175 et de $G$ candidum G48 (collection du laboratoire de génie et de microbiologie des procédés alimentaires) qui a été isolée de la surface d'un Pont l'Évêque. Cette souche (G48) est classée dans la catégorie des $G$ candidum de type levuriforme par Guéguen (1984). Sa température optimale de croissance est de $22^{\circ} \mathrm{C}$ à $25^{\circ} \mathrm{C}$ et son pH optimal est voisin de 6 . Guéguen (1984) montre également que comme toutes les souches de $G$ candidum, elle est sensible aux concentrations de sel supérieures à $5 \%$ mais qu'elle est très peu sensible aux $\mathrm{pH}$ acides et relativement peu inhibée aux températures d'af- 
finage $\left(8^{\circ} \mathrm{C}-15^{\circ} \mathrm{C}\right)$. En surface du fromage, elle se développe dès le $2^{\circ}$ jour d'affinage $\left(8\right.$ à $12^{\circ} \mathrm{C}$, $95 \%$ d'humidité relative) sous forme de cellules “ ovoïdes » de couleur crème. De plus, Lecocq et Guéguen (1994) montrent que $B$ linens ATCC 9175 se développe normalement en présence de cette souche de G candidum.

La présure à $52 \mathrm{mg} / \mathrm{L}$ de chymosine préalablement stérilisée par filtration est ajoutée à raison de $0,5 \mathrm{~mL}$ pour $200 \mathrm{~mL}$ de lait. Après caillage, moulage et égouttage, le fromage est salé en saumure saturée pendant 35 minutes. Après transfert aseptique dans une enceinte d'affinage stérile, les fromages sont ressuyés 24 heures à $80 \%$ d'humidité relative à $13{ }^{\circ} \mathrm{C}$. Ils sont ensuite affinés à $13^{\circ} \mathrm{C}$ et à $95 \%$ d'humidité relative pendant 60 jours.

\section{Détermination de la biomasse et de la quantité de pigments des cultures de $\mathrm{B}$ linens}

\section{Détermination de la concentration en cellules viables}

Le dénombrement des cellules viables est effectué avec le milieu de Piton (1988) par la méthode des dilutions successives, avec ensemencement en surface des boîtes à l'aide d'un ensemenceur spiral (Plater, Interscience, Saint-NomLa-Bretèche, France). Les boîtes ainsi ensemencées sont incubées à $25^{\circ} \mathrm{C}$ pendant 3 à 5 jours.

\section{Étude de la concentration totale des suspensions de $B$ linens en absence des fromages}

Pour chaque prélèvement, la mesure de la quantité de matière séche est réalisée par filtration de $10 \mathrm{~mL}$ de culture sur membrane en acétate de cellulose selon la procédure décrite par la norme Afnor V04-282 (1985).

La concentration totale des suspensions de $B$ linens est également déterminée par mesure de l'absorbance à $650 \mathrm{~nm}$ (Famelart et al, 1987). La relation absorbance-quantité de cellules est linéaire pour des absorbances comprises entre 0,3 et 0,7 . II en découle une légère imprécision pour les premières heures de croissance due aux faibles valeurs d'absorbance $(\leq 0,3)$. Ensuite, si nécessaire, les cultures sont diluées avec de l'eau physiologique ( $9 \mathrm{~g} / \mathrm{L}$ de $\mathrm{NaCl})$ afin de rester dans cette plage et l'absorbance de la culture est alors calculée en tenant compte du facteur de dilution.

\section{Extraction et quantification optique des pigments}

Pour les cultures en milieu liquide, la procédure d'extraction utilisée est schématisée sur la figure 1A. Elle est adaptée à partir de celles proposées par Yamaguchi (1957), Jones et al (1973), Fautz et Reinchenbach (1980) et Khol et al (1983). Cependant, contrairement à ces différents travaux, l'extraction des pigments de $B$ linens doit être quantitative. Des essais préliminaires (cultures en milieu liquide et sur fromage) ont montré que seule l'utilisation de méthanol pur (10 ou $20 \mathrm{~mL} / \mathrm{g}$ pendant $1 \mathrm{~h}$ à $25^{\circ} \mathrm{C}$ ) permettait d'extraire la totalité des pigments se trouvant dans la membrane cellulaire. Les surnageants de culture et de lavage (fig $1 \mathrm{~A})$, de même que le résidu cellulaire restant sur le filtre après extraction, ne contiennent pas de pigments. En effet, ils ne se colorent plus en rouge-carmin en présence de potasse. Grecz et Dack (1961) et Crombach (1974) ont indiqué que l'intensité de la couleur des colonies de $B$ linens est fonction de la souche, du $\mathrm{pH}$ et de la concentration en $\mathrm{NaCl}$. Pour la souche ATCC 9175 , nous avons étudié les facteurs $\mathrm{pH}$ et concentration en $\mathrm{NaCl}$. Ainsi la concentration en $\mathrm{NaCl}$ a varié de 0 à $5 \%$ (milieu à $\mathrm{pH} 7,0$ ). Pour étudier l'effet du $\mathrm{pH}$, un milieu de concentration en $\mathrm{NaCl}$ égale à $1 \%$ a été utilisé, en ajustant successivement son $\mathrm{pH}$ initial à des valeurs comprises entre 6 et 9,5. Dans tous les cas, les cultures de $50 \mathrm{~mL}$ sont réalisées en erlenmeyer de $250 \mathrm{~mL}$ et incubées pendant 48 heures. L'extrait méthanolique des pigments est alors analysé (spectrophotomètre Shimadzu UV 160) sur la plage d'absorbance $300-800 \mathrm{~nm}$ afin de déterminer la ou les longueurs d'onde à retenir pour la suite de l'étude.

Dans le cas des fromages fabriqués comme décrit précédemment, l'étude de la quantité de pigments est limitée à la surface. La croûte est prélevée selon la méthode proposée par Le Graet et Brûlé (1988). Elle est finement broyée à l'aide d'un pilon dans un mortier. Deux procédures d'extraction des pigments ont été comparées.

Premièrement, 0,5 ou $1 \mathrm{~g}$ d'échantillon finement broyé est mis en contact avec $40 \mathrm{~mL}$ d'une solution stérile de citrate à $2 \%$ et ce mélange est homogénéisé à l'aide d'un broyeur Ultra-turrax (T25, Janke Kundel) à 20500 tours/min pendant 2 minutes. La sus- 
A. Culture en milieu liquide : agitation : 250 tours/min

B. Echantillon fromage température : $25^{\circ} \mathrm{C}$ durée : 0 à $8 \mathrm{j}$

Prélèvement homogène $(40 \mathrm{ml})$

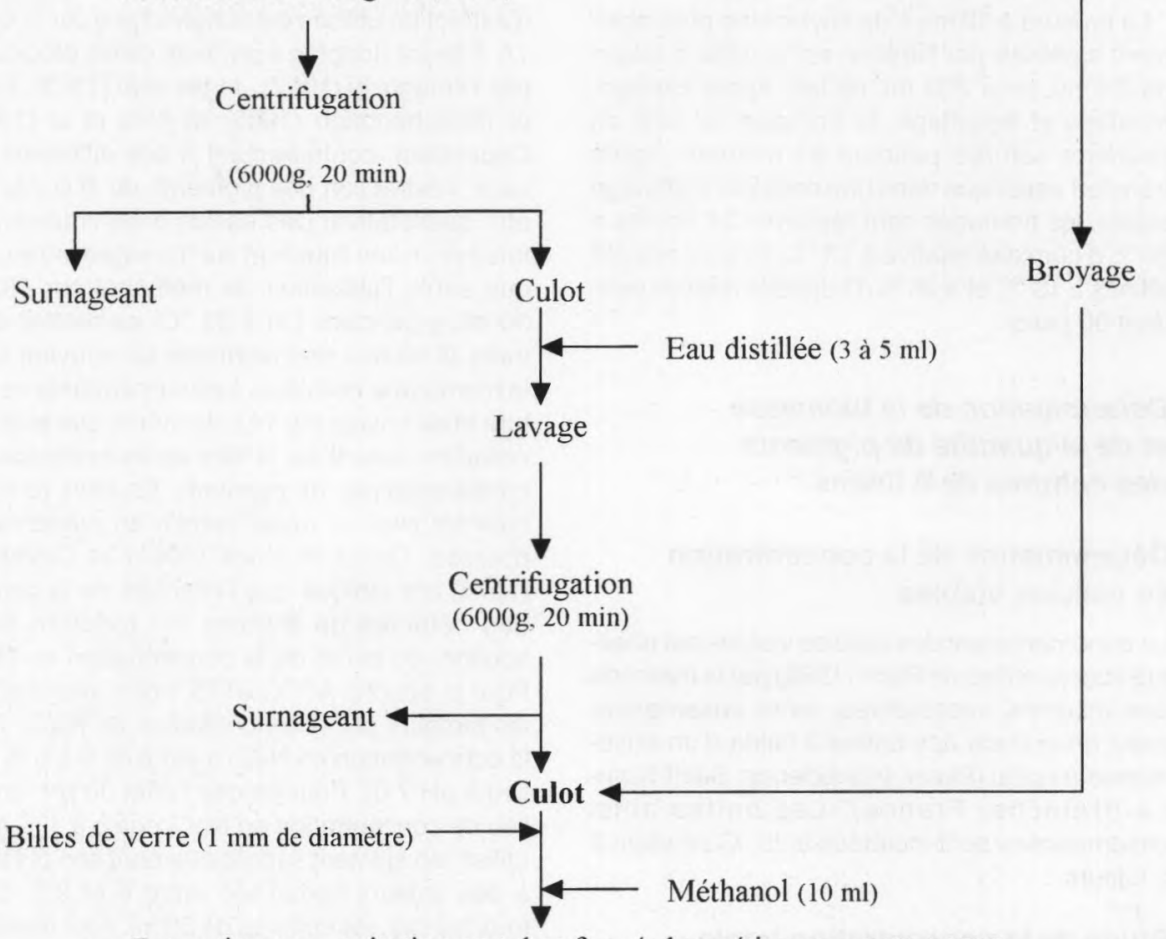

Extraction sous agitation en tubes fermés hermétiquement ( $1 \mathrm{~h}$ à $25^{\circ} \mathrm{C}$ )

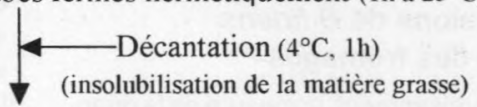

Filtration sur membrane de type RS 15 résistante au méthanol<smiles>[Te]C1CC1</smiles>

Mesure de l'absorbance -Spectre entre 300 à $800 \mathrm{~nm}$

-Absorption à $450 \mathrm{~nm}$

Fig 1. Protocole d'extraction des pigments cellulaires de $B$ linens ATCC 9175. A. Culture en milieu liquide. B. Culture en surface du fromage.

Extraction procedure of the cellular pigments of B linens ATCC 9175. A. Culture in liquid medium. B. Culture in surface of cheese. 
pension ainsi obtenue subit la procédure d'extraction décrite précédemment (fig 1B).

Deuxièmement, 0,5 ou $1 \mathrm{~g}$ d'échantillon finement broyé est directement mélangé à $10 \mathrm{~mL}$ de méthanol. Ce mélange est ensuite agité manuellement et l'extraction des pigments se fait également comme décrit sur la figure 1B. Par ailleurs, il est nécessaire de vérifier que si un constituant du fromage est solubilisé par le méthanol, il n'interfère pas sur les mesures d'absorbance de la quantité de pigments des cellules de $B$ linens. Pour cela, une culture de $B$ linens, réalisée en milieu liquide, est incubée $48 \mathrm{~h}$ à $25^{\circ} \mathrm{C}$ sous agitation continue. Elle est ensuite centrifugée à $6000 \mathrm{~g}$ pendant $20 \mathrm{~min}$ afin de récupérer l'ensemble de la biomasse. Après lavage à l'eau et centrifugation $(6000 \mathrm{~g}, 20 \mathrm{~min})$, les cellules récupérées sont divisées en trois parties égales. La première (témoin) n'est pas mélangée au fromage, la seconde est mélangée à la suspension fromagère au citrate et la dernière est directement mélangée à $1 \mathrm{~g}$ de fromage finement broyé.

Le fromage utilisé est un fromage jeune de 2 jours non ensemencé par $B$ linens. Sur chaque partie, après extraction des pigments de $B$ linens au méthanol, les extraits méthanoliques sont analysés par absorbance entre 300 et $800 \mathrm{~nm}$ afin de définir les longueurs d'ondes les mieux adaptées à la quantification des pigments en présence ou en absence de fromage. Selon ce protocole, trois cultures différentes de $B$ linens ATCC 9175 sont testées. Pour chaque échantilIon, la procédure d'extraction est réalisée en triple.

Afin de valider son application au cas des cultures de $B$ linens de surface des fromages, la méthode de dosage des pigments ainsi définie est utilisée pour suivre l'évolution de la concentration totale de $B$ linens ATCC 9175 au cours d'un affinage (durée 2 à 60 jours ; $95 \%$ d'humidité relative $; 13^{\circ} \mathrm{C}$ ).

\section{Étude statistique}

Pour chaque culture de $B$ linens en milieu liquide, les corrélations entre les quantités de cellules mesurées par pesée et par absorbance à $650 \mathrm{~nm}$ et les quantités de pigments extraits sont définies afin de vérifier que cette mesure constitue une méthode acceptable de détermination de la concentration cellulaire totale.

La linéarité de la régression linéaire biomassepigmentation est appréciée par le calcul du coefficient de corrélation au carré $\left(R^{2}\right)$. Un test de Fisher $(F)$ est appliqué pour évaluer l'égalité des régressions linéaires des différents essais (Vessereau, 1988). Ce test permet de comparer deux populations soumises à l'hypothèse suivante : égalité des régressions linéaires entre les différents essais. Chaque régression linéaire génère un modèle (indice i) caractérisé par la somme des écarts au carré (SS) et par son degré de liberté $\left(d_{l}\right)$. Pour cette hypothèse, la distribution $F$ est calculée avec des degrés de liberté $\left(d l_{0}-d l_{i}\right)$ pour le numérateur et $d l_{0}$ pour le dénominateur selon la relation 1 :

$$
F=\frac{\left(\frac{S S_{0}-\sum_{i=1}^{n} S S_{i}}{d l_{0}-\sum_{i=1}^{n} d l_{i}}\right)}{\left(\frac{S S_{0}}{d l_{0}}\right)}
$$

où $d l_{0}$ et $S S_{0}$ représentent respectivement le degré de liberté et la somme des écarts au carré des populations prises ensemble.

Les niveaux de confiance de $95 \%$ et $99 \%$ sont testés. L'hypothèse est acceptée si la distribution $F$ calculée est plus petite que la valeur donnée dans les tables de Fisher-Snedecor pour le niveau de confiance de $95 \%$ ou $99 \%$ (Dagnelie, 1992). L'hypothèse est probablement erronée si la distribution $F$ calculée est voisine des valeurs données dans les tables de Fisher-Snedecor à des niveaux de confiance de $99 \%$ et de $95 \%$. L'hypothèse est considérée comme très certainement erronée dans les autres cas.

\section{RÉSULTATS}

\section{Détermination de l'absorbance maximale, effet $\mathrm{du} \mathrm{pH}$ et de la concentration en $\mathrm{NaCl}$ sur la pigmentation}

\section{Suspensions de $B$ linens en absence de fromage}

Les différents spectres d'absorbance des extraits méthanoliques des pigments cellulaires présentent systématiquement deux pics d'absorbance importante: l'un mineur à $392 \mathrm{~nm}$ et l'autre majeur à $450 \mathrm{~nm}$ (fig 2). Les absorbances à l'une et l'autre de ces 
deux longueurs d'onde pourraient servir pour apprécier la quantité de cellules. La longueur d'onde $450 \mathrm{~nm}$ est retenue pour la suite de l'étude. En outre, sachant que la totalité des pigments extraits par le méthanol est mesurée par absorbance à $450 \mathrm{~nm}$, cette mesure est assimilée à la quantité de pigments produite par $B$ linens.

Les essais réalisés afin d'étudier l'influence $\mathrm{du} \mathrm{pH}$ et de la concentration en $\mathrm{NaCl}$ du milieu de culture sur l'absorbance permettent de conclure que ces facteurs n'ont pas d'influence. En effet, après extraction des pigments des cellules, les spectres d'absorbance des extraits méthanoliques présentent les mêmes pics caractéristiques, quelle que soit la concentration en $\mathrm{NaCl}$ utilisée ou quel que soit le $\mathrm{pH}$ initial du milieu. De plus, la croissance de $B$ linens est maximale lorsque le $\mathrm{pH}$ initial est de 7,5 et nulle à un pH inférieur à 6,5 (résultat attendu). En revanche, aucune différence significative n'est mise en évidence quand la concentration en $\mathrm{NaCl}$ ajouté varie de 0 à $5 \%$, valeur en rapport avec celle existant dans la phase aqueuse d'un fromage.

\section{Suspensions de $B$ linens en présence de fromage}

Dans le cas où le fromage est préalablement mis en suspension dans du citrate à $2 \%$ avant d'être additionné d'une quantité connue de $B$ linens, la solution obtenue après extraction des pigments n'est pas limpide. L'analyse de la quantité de pigments par absorbance s'avère impossible.

Dans le cas où le fromage est directement mélangé à une quantité connue de $B$ linens, la solution obtenue après extraction des pigments au méthanol est parfaitement limpide. Cependant, lorsque la quantité de fromage utilisée est supérieure à $0,5 \mathrm{~g}$ pour $10 \mathrm{~mL}$ de méthanol, la totalité des pigments n'est pas extraite.

En effet, le culot est encore orange et réagit avec l'hydroxyde de potassium (KOH 5N) (Jones et al, 1973). Pour éviter cela, dans la suite de l'étude, l'extraction des pigments de $B$ linens se fera sur $0,5 \mathrm{~g}$ de croûte pour $10 \mathrm{~mL}$ de méthanol. Dans ces conditions, les spectres d'absorption des extraits méthanoliques de $B$ linens en

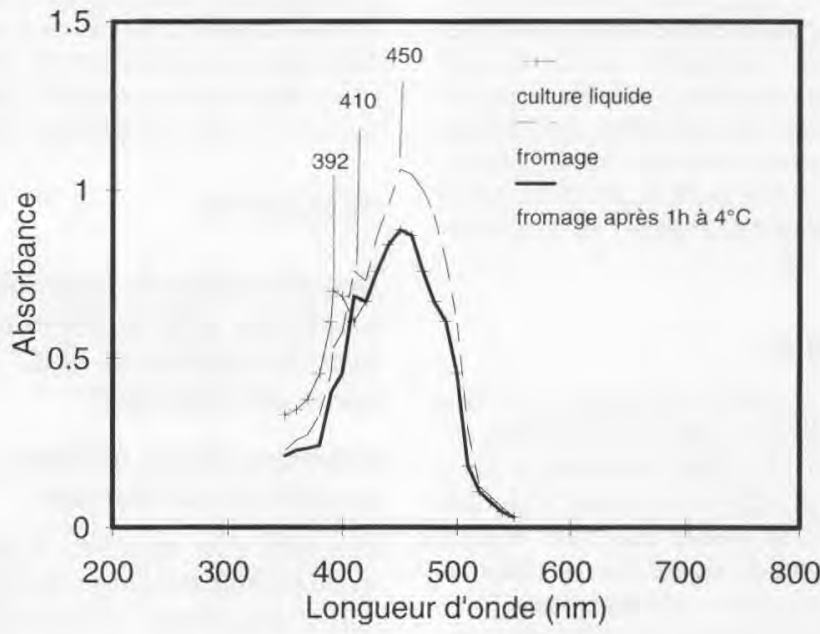

Fig 2. Spectre d'absorption des pigments extraits dans le méthanol. Absorption spectra of pigment extract in methanol. 
Tableau I. Résultats des tests statistiques relatifs aux régressions linéaires établies entre la quantité de pigments cellulaires et la concentration cellulaire totale mesurée par pesée ou par absorbance.

Statistical F-test applied to the linear regression established between the cellular pigment quantity and the total $\mathrm{B}$ linens concentration measured by dry weight or by absorbance at $650 \mathrm{~nm}$.

\begin{tabular}{|c|c|c|c|c|c|c|c|c|}
\hline $\begin{array}{c}\text { Variable } \\
Y\end{array}$ & $\begin{array}{c}\text { Variable } \\
x\end{array}$ & & $R^{2}$ & $F$ & $d l_{0}-\Sigma d l_{i}$ & $d l_{0}$ & Test-F & $S$ \\
\hline \multirow{4}{*}{$P S(g / L)$} & A 650 & $\begin{array}{l}\text { totalité de } \\
\text { la culture }\end{array}$ & 0,94 & 3,89 & 1 & 63 & + & 0,3 \\
\hline & & $\begin{array}{l}\text { phase de } \\
\text { croissance }\end{array}$ & 0,86 & 18 & 1 & 29 & - & 0,3 \\
\hline & A 450 & $\begin{array}{l}\text { totalité de } \\
\text { la culture }\end{array}$ & 0,86 & 11,3 & 1 & 62 & - & 0,2 \\
\hline & & $\begin{array}{l}\text { phase de } \\
\text { croissance }\end{array}$ & 0,70 & 37 & 1 & 29 & - & 0,1 \\
\hline \multirow[t]{2}{*}{ A 650} & A 450 & $\begin{array}{l}\text { totalité de } \\
\text { la culture }\end{array}$ & 0,90 & 2,1 & 1 & 63 & ++ & 0,3 \\
\hline & & $\begin{array}{l}\text { phase de } \\
\text { croissance }\end{array}$ & 0,88 & 6,50 & 1 & 30 & + & 0,01 \\
\hline
\end{tabular}

PS : quantité de matière sèche $(\mathrm{g} / \mathrm{L}$ ), A 650 : absorbance à $650 \mathrm{~nm}, \mathrm{~A} 450$ : quantité de pigments (absorbance à $450 \mathrm{~nm}), \mathrm{R}$ : coefficient de corrélation, $F$ : valeur du test de Fisher, dlo: degré de liberté de l'ensemble des cultures, $d l_{i}$ : degré de liberté de la culture $\mathrm{i},(++)$ : hypothèse acceptée à $95 \%,(+)$ : hypothèse acceptée à $99 \%,(-)$ : hypothèse rejetée, phase de croissance : temps de culture inférieur à $60 \mathrm{~h}$, totalité de la culture : temps de culture égal à $150 \mathrm{~h}$, $S$ : seuil de détection exprimé en $\mathrm{g} / \mathrm{L}$ ou en unité d'absorbance à $650 \mathrm{~nm}$.

PS: dry matter quantity $(\mathrm{g} / \mathrm{L})$, A 650: absorbance at $650 \mathrm{~nm}$, A 450: absorbance at $450 \mathrm{~nm}$ (pigment quantity), $R$ : correlation coefficient, F: value of F-test, dlo: degree of freedom for all cultures, dli: degree of freedom for culture $i$, $(++)$ : hypothesis accepted at the $95 \%,(+)$ : hypothesis accepted at the $99 \%,(-)$ : hypothesis rejected, growth's phase: culture time inferior to $60 \mathrm{~h}$, culture's totality: culture time equal to $150 \mathrm{~h}, \mathrm{~S}$ : threshold of detection.

présence de fromage présentent deux pics à $410 \mathrm{~nm}$ et $450 \mathrm{~nm}$ (fig 2). II y a sûrement interaction entre les pigments et la matière grasse pour le premier pic.

Par ailleurs, il est remarqué que la matière grasse des fromages solubilisée par le méthanol entraîne une surestimation de la quantité de pigments observée à $450 \mathrm{~nm}$. Pour éviter cela, les extraits méthanoliques sont incubés à $4{ }^{\circ} \mathrm{C}$ pendant 1 heure avant de réaliser la filtration et la mesure de l'absorbance (fig 2).

Dans ces conditions, la quantité de pigments mesurée par absorbance à $450 \mathrm{~nm}$ en présence de fromage est identique, aux erreurs près, à celles de l'échantillon témoin.

Par la suite, la quantité de pigments des cellules de $B$ linens, quel que soit le milieu de culture utilisé, sera déterminée par une mesure d'absorbance à $450 \mathrm{~nm}$.

\section{Analyse de la croissance de B linens sur milieu liquide}

Un exemple d'évolution de la quantité de matière sèche (PS), de la concentration en cellules viables (UFC), de l'absorbance à $650 \mathrm{~nm}$ et de la quantité de pigments (c'est-à-dire de l'absorbance à 450 nm) en 


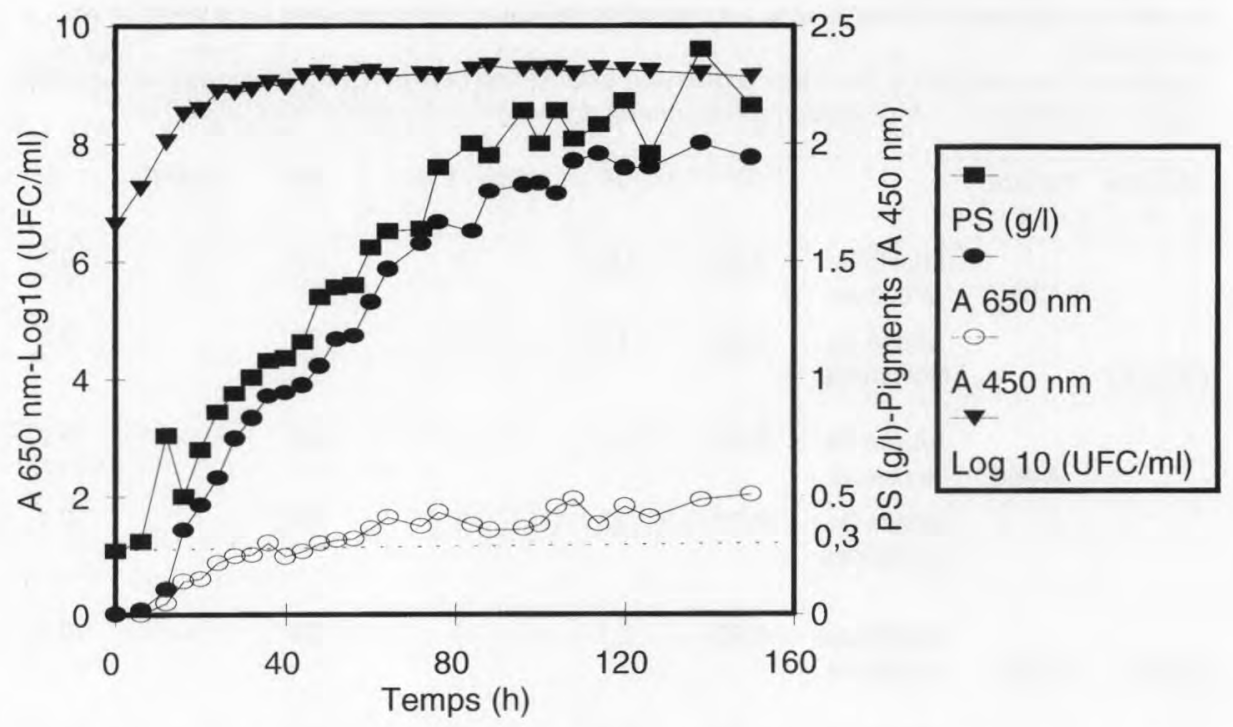

Fig 3. Exemple d'évolution de la quantité de matière sèche PS ( $g / L)$, de l'absorbance A $650 \mathrm{~nm}$ (A 650), de la concentration en cellules viables (UFC/mL) et de la quantité de pigments mesurée par absorbance A $450 \mathrm{~nm}$ (A 450) d'une culture de $B$ linens en fonction du temps d'incubation $\left(25^{\circ} \mathrm{C}\right.$, milieu liquide, agitation : 250 tours/min).

Evolution of dry matter PS ( $g / L$ ), absorbance at $650 \mathrm{~nm}$ (A 650), viable cell concentration (UFC/mL) and pigment quantity measured by absorbance at $450 \mathrm{~nm}$ ( $A$ 450) of a culture of $\mathrm{B}$ linens as a function of fermentation time $\left(25^{\circ} \mathrm{C}\right.$; liquid medium, agitation: $\left.250 \mathrm{rpm}\right)$.

Tableau II. Évolution de la concentration cellulaire totale de $B$ linens mesurée par absorbance à $450 \mathrm{~nm}$ en fonction de la durée d'affinage dans le cas d'un fromage expérimental.

Evolution of total cell concentration of B linens obtained by absorbance at $450 \mathrm{~nm}$ in relation to ripening time for an experimental bacterial surface-ripened cheese.

\begin{tabular}{cccc}
\hline Durée d'affinage $(j)$ & $\begin{array}{c}\text { Couleur observée, } \\
\text { à l'oil } n u\end{array}$ & $\begin{array}{c}\text { Absorbance à } \\
450 \mathrm{~nm}\end{array}$ & $\begin{array}{c}\text { Bactéries viables } \\
\text { (UFC/g de croûte) }\end{array}$ \\
\hline 2 & blanc & 0 & $4,410^{6}$ \\
6 & blanc & 0 & $6,510^{7}$ \\
14 & blanc & 0 & $2,310^{7}$ \\
20 & crème & 0 & $4,010^{8}$ \\
23 & crème & 0,01 & $3,010^{9}$ \\
29 & crème & 0,08 & $2,310^{11}$ \\
35 & orange & 0,17 & $4,010^{10}$ \\
45 & orange & 0,54 & $4,310^{10}$ \\
60 & orange & 0,55 & $3,710^{10}$ \\
\hline
\end{tabular}


fonction du temps d'incubation est présenté à la figure 3 . Sur un plan général, l'ensemble des résultats expérimentaux permettent de constater que :

- l'évolution de la quantité de la matière sèche en fonction du temps d'incubation est analogue à celle de l'absorbance à $650 \mathrm{~nm}$; cependant, pour les premières heures de culture, les valeurs d'absorbance à $650 \mathrm{~nm}$ et surtout celles de matière sèche sont imprécises, en raison des faibles concentrations cellulaires ;

- en fin de culture, les concentrations cellulaires sont voisines de $2 \mathrm{~g} / \mathrm{L}$ pour la matière sèche, ce qui correspond à 8 pour l'absorbance à $650 \mathrm{~nm}$;

- l'évolution de la concentration en cellules viables en fonction du temps de culture se divise en trois phases : la première est une phase de latence et d'initiation de la croissance (temps d'incubation inférieur à $16 \mathrm{~h}$ ), la seconde est une phase de croissance active (temps d'incubation compris entre 16 heures et 60 heures environ), la dernière est une phase stationnaire qui débute aux environs de la $60 \mathrm{e}$ heure de culture; au-delà de la centième heure de culture, le nombre de cellules viables commence à décroître ;

- la quantité de pigments mesurée par absorbance à $450 \mathrm{~nm}$ suit la même évolution que celle observée pour la quantité de matière sèche ou l'absorbance à $650 \mathrm{~nm}$. Elle ne diminue pas lorsque certaines cellules

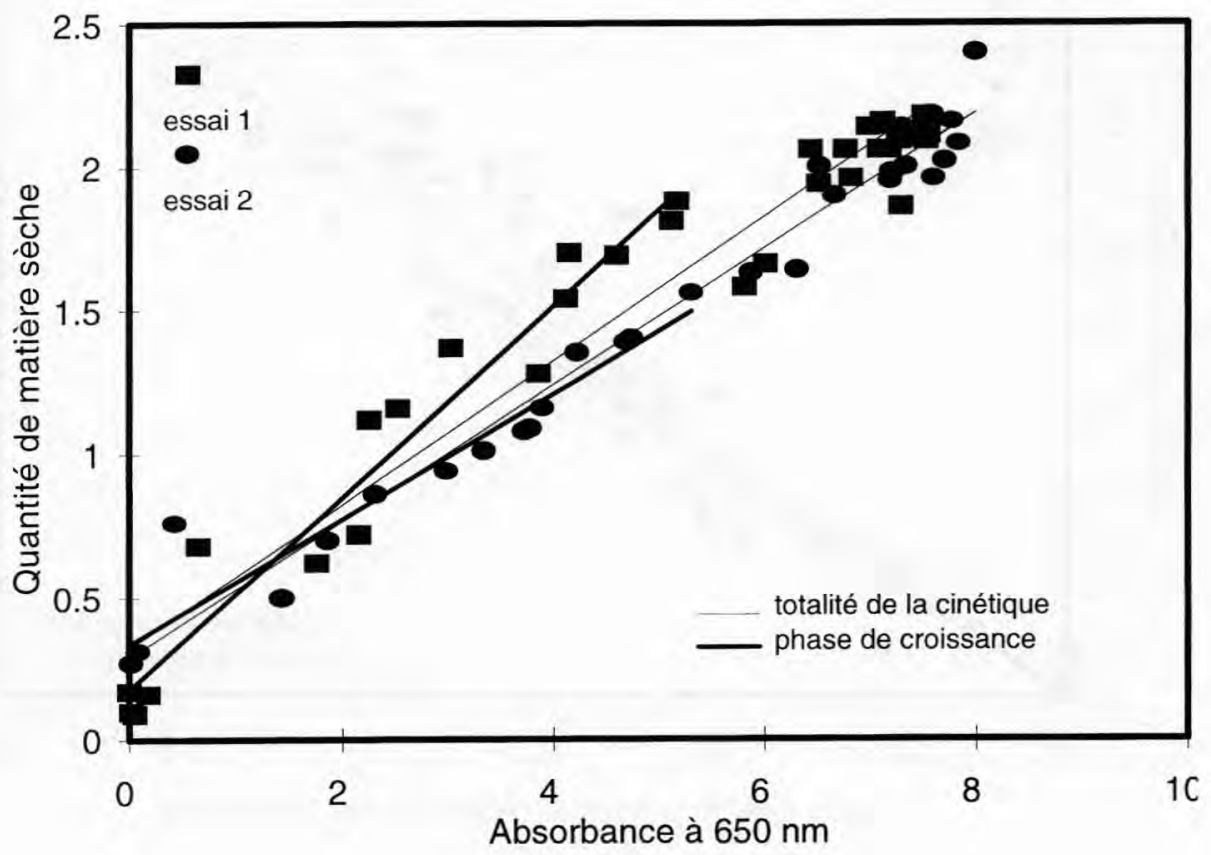

Fig 4. Étude des corrélations linéaires existant entre la quantité de matière sèche ( $\mathrm{g} / \mathrm{L}$ ) et l'absorbance à $650 \mathrm{~nm}$.

Linear correlations established between dry matter $(\mathrm{g} / \mathrm{L})$ and absorbance at $650 \mathrm{~nm}$. 
de $B$ linens deviennent inactives (légère réduction du nombre de UFC/mL après la 100 e heure).

\section{Corrélations entre les méthodes de mesure de la concentration cellulaire}

\section{Corrélation entre la quantité de matière sèche et l'absorbance à $650 \mathrm{~nm}$}

Les résultats présentés dans le tableau I et la figure 4 permettent de constater que : - aucune différence significative de relation n'est obtenue entre les deux essais lorsque la relation est étudiée sur l'ensemble de la cinétique (temps d'incubation de 8 jours) ;
- la linéarité de la relation est satisfaisante $\left(R^{2} \geq 0,94\right)$ sur la totalité de la cinétique, quel que soitl'essai considéré ; la pente et l'ordonnée à l'origine de la régression sont indépendantes de l'essai puisque l'hypothèse d'égalité des régressions (Test-F) est vérifiée ; - quel que soit l'essai considéré, la relation 2 traduisant la quantité de matière sèche (PS ; g/L) en fonction de l'absorbance à $650 \mathrm{~nm}\left(A_{650}\right)$ s'écrit :

$$
P S=0,3+0,24 . A_{650}
$$

- il existe un seuil minimal de matière sèche en dessous duquel aucune estimation de l'absorbance à $650 \mathrm{~nm}$ n'est possible ; ce seuil est de $0,3 \mathrm{~g} / \mathrm{L}$;

- Iorsque l'étude est limitée à la seule

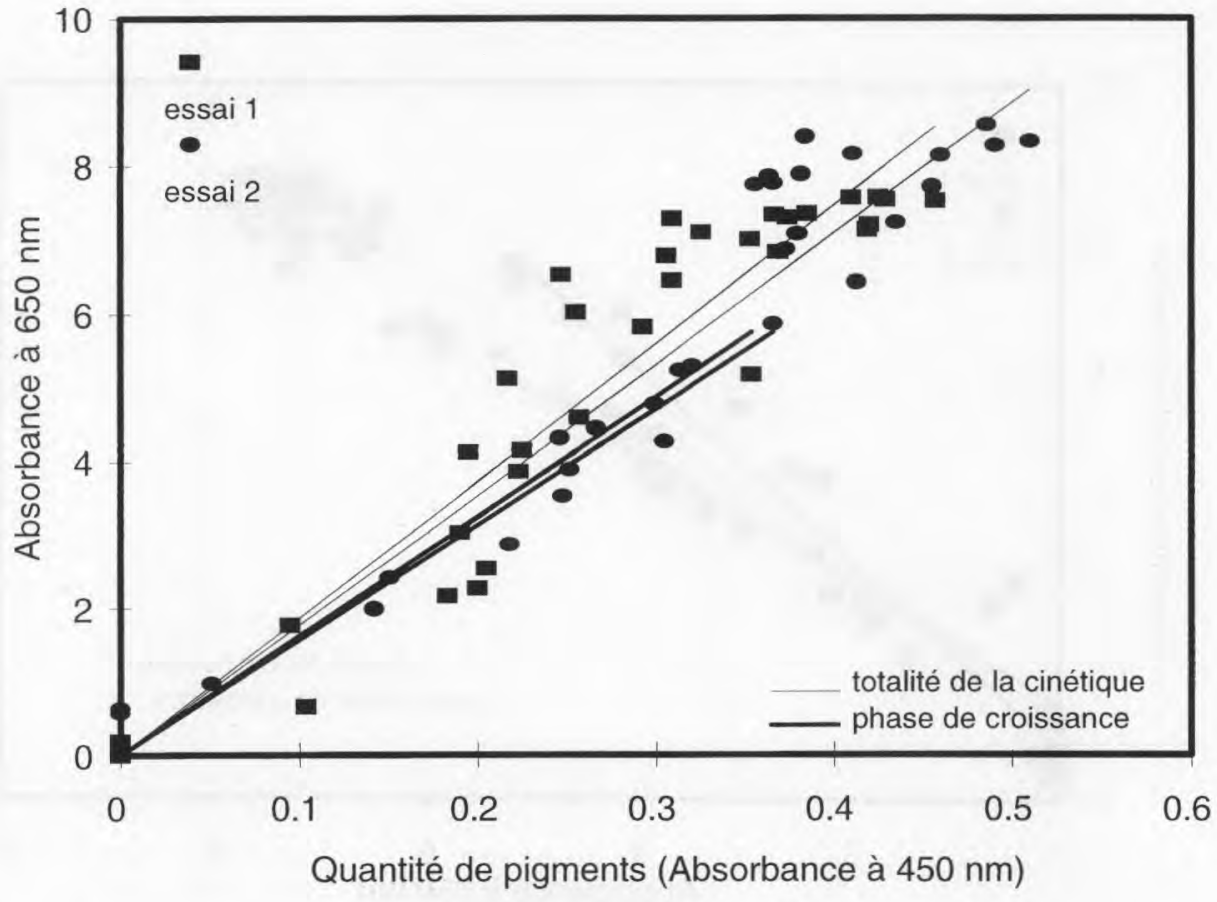

Fig 5. Étude des corrélations linéaires existant entre la quantité de pigments ('’absorbance à $450 \mathrm{~nm}$ ) et l'absorbance à $650 \mathrm{~nm}$.

Linear correlations established between pigment quantity (absorbance at $450 \mathrm{~nm}$ ) and absorbance at $650 \mathrm{~nm}$. 
phase de croissance, bien que la linéarité des relations individuelles soit acceptable $\left(R^{2} \geq 0,86\right)$, l'hypothèse d'égalité des essais n'est plus vérifiée.

\section{Corrélation entre la quantité de matière sèche et la quantité de pigments}

Les résultats présentés dans le tableau I permettent de constater que si l'on considère la totalité de la culture, bien que la linéarité des régressions de chaque essai soit acceptable $\left(R^{2} \geq 0,86\right)$, I'hypothèse d'égalité des essais n'est jamais satisfaite. De plus, il existe un seuil minimum de détection de la quantité de matière cellulaire sèche de $0,2 \mathrm{~g} / \mathrm{L}$.

De plus, lorsque l'étude de la corrélation est limitée à la phase de croissance, la linéarité des deux essais pris individuellement ou ensemble est mauvaise ( $R 2=0,70$ ) et les deux essais sont statistiquement différents.

\section{Corrélation entre l'absorbance à $650 \mathrm{~nm}$ et la quantité de pigments}

Les résultats présentés dans le tableau I et dans la figure 5 montrent que lorsque la culture est considérée dans son ensemble, la linéarité de la corrélation est bonne $\left(R^{2} \geq 0,90\right)$. Le test $F$ étant vérifié à un seuil de probabilité de 0,05 , les deux essais sont identiques. La régression reliant les absorbances à $650 \mathrm{~nm}$ et à $450 \mathrm{~nm}$ correspond alors à la relation 3 . Cette relation montre un seuil de détection minimal en absorbance à $650 \mathrm{~nm}$ de 0,3 .

$$
A_{650}=-0,30+18,1 \cdot A_{450}
$$

Les mêmes observations sont faites lorsque l'étude de la corrélation entre l'absorbance à $650 \mathrm{~nm}$ et la quantité de pigments est limitée à la phase de croissance. La régression ainsi obtenue correspond à la relation 4 :

$$
A_{650}=-0,01+14,73 . A_{450}
$$

\section{Application de la méthode de dosage des pigments de $\mathrm{B}$ linens au fromage}

Les résultats de mesure de la concentration totale en $B$ linens à la surface de fromages affinés entre 2 jours et 60 jours font l'objet du tableau II. Chaque résultat rapporté (absorbance à $450 \mathrm{~nm}$ et concentration en cellules viables) est la moyenne de trois déterminations. La coloration des fromages par $B$ linens n'est observée à l'œil nu qu'à partir du $20^{\circ}$ jour d'affinage. Cela est confirmé par étude des mesures d'absorbance à $450 \mathrm{~nm}$, qui traduisent la quantité de pigments de $B$ linens. Plus la couleur crème-orange de la croûte du fromage (observée à l'œil nu) est intense, plus la quantité de pigments est élevée.

La concentration en cellules viables de $B$ linens est de $4,410^{6} \mathrm{UFC} / \mathrm{mL}$ à 2 jours. Elle augmente régulièrement à partir du $15^{e}$ jour jusqu'à atteindre $2,310^{11} \mathrm{UFC} / \mathrm{mL}$ vers le $29^{e}$ jour d'affinage et diminue progressivement par la suite. En revanche, la quantité de pigments augmente avec la durée d'affinage au moins jusqu'au $45^{\circ}$ jour. Cela pourrait s'expliquer en supposant que la quantité totale de cellules de $B$ linens continue d'augmenter alors que le maximum de cellules viables a été atteint et commence même à décroître.

\section{DISCUSSION}

La pigmentation orange des colonies de $B$ linens est un critère taxonomique reconnu (Grecz et Dack, 1961). Mais, à notre connaissance, les études concernant les pigments cellulaires de $B$ linens ont été purement analytiques et qualitatives (Jones et al, 1973 ; Khol et al, 1983). Elles n'ont pas été utilisées jusqu'à maintenant comme moyen de mesure de la croissance de $B$ linens, même en culture pure. Même l'influence de différents facteurs déjà observés (lumière, $\mathrm{pH}, \mathrm{NaCl}$...) n'a pas été étudiée d'un point de vue quantitatif. Notre étude permet de montrer que la quantité de 
pigments produits par la souche de $B$ linens ATCC 9175 est indépendante de la concentration en $\mathrm{NaCl}$ et/ou du $\mathrm{pH}$ du milieu de culture utilisé. Cette observation est en accord avec celle de Crombach (1974) dans le cas de concentrations en $\mathrm{NaCl}$ inférieures à $4 \%$.

Selon la procédure d'extraction utilisée, les pigments sont totalement extraits de la membrane des cellules lorsque le méthanol est utilisé pur à $25^{\circ} \mathrm{C}$. Dès lors, la potasse $(5 \mathrm{~N})$ n'agit plus sur le culot d'extraction, comme cela a été montré par Grecz et Dack (1961) et Jones et al (1973).

Le même résultat a été obtenu lors du traitement de bactéries en surface de fromage ; cela laisse supposer que le méthanol agit de façon similaire sur les pigments cellulaires de $B$ linens, même si une partie de la matière grasse du fromage est extraite en même temps que les pigments.

La valeur du pic d'absorbance à $450 \mathrm{~nm}$ traduit correctement la quantité de pigments extraits des cellules de $B$ linens. En effet, l'étude de l'absorbance de l'extrait méthanolique des pigments cellulaires des cultures de $B$ linens ATCC 9175 en milieu liquide permet de définir deux longueurs d'onde pour lesquelles l'absorbance présente un pic caractéristique : $392 \mathrm{~nm}$ et $450 \mathrm{~nm}$.

Ces résultats sont en accord avec ceux de Jones et al (1973), qui montrent que les pics d'absorbance des extraits méthanoliques sont fonction de la souche de bactérie corynéforme étudiée mais restent situés entre $360 \mathrm{~nm}$ et $480 \mathrm{~nm}$ et sont, en général au nombre de deux ou trois par souche. Avec la même souche de $B$ linens que celle utilisée ici (ATCC 9175), Khol et al (1983) ont montré l'existence de trois pigments. Dans le méthanol, ces derniers présentent une couleur jaune-orange et le même pic d'absorbance (454 nm).

Le pic majeur que nous obtenons se trouve à peu près à la même longueur d'onde $(450 \mathrm{~nm})$ que celui de Khol et al (1983), mais nous en obtenons un deuxième, moins important, à $392 \mathrm{~nm}$.
Deux hypothèses peuvent expliquer cette différence : soit Khol et al (1983) ne l'ont pas mentionnée (en effet, Jones et al [1973] trouvent également un second pic à $400 \mathrm{~nm}$ pour deux autres souches de $B / \mathrm{l}$ nens), soit elle est due à la différence de composition des milieux de culture utilisés. D'ailleurs, l'influence de la composition du milieu sur la pigmentation permettrait également d'expliquer la différence entre les pics observés sur le milieu liquide et les fromages. En effet, bien que la composition du milieu liquide simule celle d'un fromage jeune, elle n'est pas strictement identique et ne comporte pas de matière grasse. De plus, la matière grasse du fromage extraite par le méthanol interfère directement sur les mesures d'absorbance dans la plage 300-420 nm, même lorsque le protocole expérimental permet d'en éliminer une grande partie.

En ce qui concerne l'âge de la culture, deux cas ont été considérés : soit la phase de croissance seule (temps de culture inférieur à 60 heures) soit l'ensemble de la culture (150 heures). Pour chaque essai, la corrélation linéaire reliant l'absorbance à $650 \mathrm{~nm}$ et la quantité de matière sèche est bonne $\left(R^{2}>0,9\right)$ que l'étude soit réalisée sur l'ensemble de la cinétique ou seulement sur la phase de croissance. Cependant, comme le montre le test de Fisher, cette corrélation n'est pas significativement indépendante de l'essai. Cela s'explique en partie par l'imprécision des valeurs d'absorbance à $650 \mathrm{~nm}$ (Kouomegne et al, 1984) et surtout, des pesées pour les faibles concentrations bactériennes (Guiraud et Galzy, 1980). De plus, comme le supposent Famelart et al (1987), la quantité de matière sèche peut être surestimée par la production de polysaccharides associée au développement de $B$ linens.

En ce qui concerne la relation entre les quantités de pigments et de matière sèche, la corrélation linéaire obtenue pour chaque culture est satisfaisante $\left(R^{2}>0,86\right)$ tant pour la phase de croissance que pour l'en- 
semble de la culture. Cependant, la relation linéaire obtenue est également dépendante de l'essai, probablement pour les mêmes raisons que celles évoquées ci-dessus à propos de la détermination de la matière sèche.

En revanche, en ce qui concerne la relation entre la quantité de pigments et l'absorbance à $650 \mathrm{~nm}$, la corrélation linéaire obtenue est satisfaisante ( $R 2 \geq 0,9)$ et indépendante de la culture considérée. Celai est vrai que la culture soit limitée à la phase de croissance ou considérée dans son ensemble. Cependant, les relations obtenues ne sont vérifiées qu'au dessus d'un seuil de détection de 0,3 unité d'absorbance à $650 \mathrm{~nm}$. Au-delà de ce seuil, la quantité de pigments des extraits méthanoliques permet donc de mesurer la concentration totale en cellules de $B$ linens.

Dans le cas des fromages, l'extrait méthanolique des pigments de $B$ linens est réalisé sur $0,5 \mathrm{~g}$ de croûte. Pour extraire les matières grasses de ce type de produits, on utilise couramment le méthanol. Mais, lors de l'extraction des pigments de $B$ linens en présence de fromage, une partie de la matière grasse du produit est également solubilisée ; ce qui provoque une interaction lors des mesures d'absorbance à $450 \mathrm{~nm}$. Pour éviter cette interaction, les extraits méthanoliques sont placés 1 heure à $4{ }^{\circ} \mathrm{C}$ avant filtration à froid, afin d'insolubiliser les matières grasses du fromage. Dans ces conditions, le pic d'absorption à $450 \mathrm{~nm}$ de la solution méthanolique est le même pour les fromages que pour les cultures en milieu liquide.

L'étude de la concentration totale en $B$ linens, par mesure de la quantité de pigments en fonction de la durée d'affinage, est réalisée sur une série de fromages affinés de 2 à 60 jours. Pendant les 3 premieres semaines de maturation, la concentration totale en cellules de $B$ linens est relativement faible. Elle croît ensuite régulièrement jusqu'au $60^{\mathrm{A}}$ jour d'affinage. La concentration en cellules viables passe par un maximum au $29^{\mathrm{e}}$ jour d'affinage, comme cela a été observé dans le cas du Pont-l'Évêque par Daluzeau (1986). La technique mise au point s'avère donc utile pour étudier les évolutions de concentrations microbiennes et les cinétiques correspondantes.

Cependant, dans le cas de fromages comportant une flore d'affinage plus complexe, la mesure de la quantité de pigments (absorbance à $450 \mathrm{~nm}$ ) risque de représenter non seulement les pigments de $B$ linens mais l'ensemble des pigments des micro-organismes extractibles par le méthanol et capables d'absorber la lumière à $450 \mathrm{~nm}$.

En conséquence, la méthode mise au point n'est utilisable que dans le cas de fromages ensemencés avec une flore d'affinage bactérienne limitée à $B$ linens ATCC 9175 , seule ou en présence d'autres bactéries non pigmentées. Dans le cas de l'ensemencement avec d'autres souches de $B$ linens, dont la pigmentation ne dépend pas de la lumière, il serait simplement nécessaire de vérifier certains des résultats obtenus avec ATCC 9175. Pour les autres bactéries pigmentées, l'ensemble de l'étude serait à reprendre,

\section{RÉFÉRENCES}

Afnor NF 04-282 (1985) Fromages et fromages fondus, détermination de la matière sèche (méthode de référence). In : Contrôle de la qualité des produits laitiers, analyses physiques et chimiques. (S Amariglio, ed), $3^{\circ}$ édn, Tec \& Doc Lavoisier, Paris, 581-585

Bourgeois CM, Mafart P (1991) Techniques globales d'évaluation de la microflore. In: Techniques d'analyses et de contrôle dans les industries agro-alimentaires, vol 3, Le contrôle microbiologique (Bourgeois CM, Leveau JY, éds). Tec \& Doc Lavoisier APRIA, Paris, 50-71

Boyaval P, Desmazeaud MJ (1983) Le point des connaissances sur Brevibacterium linens. Lait 63, 187. 216

Crombach WHJ (1974) Morphology and physiology of coryneform bacteria. Antonie van Leeuwenhoek 40, 361-376

Dagnelie P (1992) Les tests d'hypothèses. In : Statistiques théoriques et appliquées, tome 1. Presses agronomiques de Gembloux, Gembloux, 415-444

Dagonneau H (1979) Étude des acides gras et des hydrocarbures de Brevibacterium linens. Recherche de ces composés dans quelques fromages. Thèse de $3^{e}$ cycle. Université de Paris XI-ENSIA, $81 \mathrm{p}$ 
Daluzeau D (1986) Le livarot. Thèse de docteur vétérinaire, École nationale vétérinaire de Toulouse, $76 p$

Driehuis F, Teernstra EJM (1992) Method for turbidimetric estimation of lactic acid bacteria in fat-containing milk. Neth Milk Dairy J 46, 209-215

Famelart MH, Kobilinsky A, Bouillanne C, Desmazeaud MJ (1987) Influence of temperature, $\mathrm{pH}$ and dissolved oxygen on growth of Brevibacterium linens in a fermentor. Appl Microbiol Biotechnol 25, 442448

Fautz E, Reinchenbach H (1980) A simple test for flexirubin-type pigment. FEMS Microbiol Lett 8, 87-91

Grecz N, Dack GM (1961) Taxonomically significant color reactions of Brevibacterium linens. J Bacteriol $82,241-246$

Guéguen M (1984) Contribution à la connaissance de Geotrichum candidum et notamment de sa variabilité. Conséquences pour l'industrie fromagère. Thèse de docteur d'état es sciences, Université de Caen, $471 \mathrm{p}$

Guiraud J, Galzy P (1980) L'examen au microscope. I. Techniques générales de la microbiologie. In : L'analyse microbiologique dans les industries alimentaires, Éditions de I'Usine Nouvelle, Paris, Collection Génie alimentaire, 11-29

Hadley WK, Waldman F, Fulwyler M (1985) Rapid microbiological analysis by flow cytometry. In : Instrument methods for rapid microbiological analysis (Nelson W, ed). Elsevier, New-York, 67-89

Jones D, Watkins J, Erickson SK (1973) Taxonomically significant color changes in Brevibacterium linens. Probably associated with a carotenoid-like pigment. J Gen Microbiol 77, 145-150

Khol W, Achenbach H, Reichenbach H (1983) The pigments of Brevibacterium linens: aromatic carotenoids. Phytochemistry 22, 207-210
Kouomegne R, Bracquart P, Linden G (1984) Application d'un réactif de transparisation du lait au dénombrement de bactéries. Lait 64, 418-435

Le Graet Y, Brûlé G (1988) Migration des macro et oligoéléments dans un fromage à pâte molle de type Camembert. Lait 68, 219-234

Leclercq-Perlat MN, Bergère JL, Corrieu G (1995) Mise au point d'une méthode de dénombrement de la totalité des cellules de levures de la surface d'un fromage à pâte molle. Lait $75,151-168$

Lecocq J, Guéguen M (1994) Effect of $\mathrm{pH}$ and sodium chloride on the interaction between Geotrichum candidum and Brevibacterium linens. J Dairy Sci 77 , 2890-2899

Lenoir J (1991) Deux exigences : I'hygiène et le goût. Rev Lait Fr 49, 5-6

Lenoir J, Lamberet G, Schmidt JL (1983) L'élaboration d'un fromage : l'exemple du Camembert. Pour Sci $69,30-42$

Lenoir J, Lamberet G, Schmidt JL, Tourneur C (1985) La maîtrise du bioréacteur fromage. Biofutur 41, 23-50

Miozzari GF, Niederberger P, Hutter R (1978) Permeabilization of microorganisms by Triton $X 100$. Anal Biochem 90, 220-223

Mulder EG, Adams AD, Antheunisse J, Deinema MH, Woldendors JW, Zevenhuizen LPTM (1966) The relationship between Brevibacterium linens and bacteria of genus Arthrobacter. J Appl Bacterio/ 29, 44-71

Piton C (1988) Évolution de la flore microbienne de surface de gruyère de Comté au cours de l'affinage. Lait 68, 419-434

Vessereau A (1988) Méthodes statistiques en biologie et en agronomie. Test de comparaison de moyennes et de variances. (Bailliere JB, éd), Tec et Doc Lavoisier, Paris, 134-158

Yamaguchi M (1957) On carotenoids of sponge a $R e$ niera japonica ". Bull Chem Soc Jpn 30, 111-114 